\title{
AN APPROACH FOR ASSESSING DESIGN SYSTEMS Design System Simulation and Analysis for Performance Assessment
}

\author{
Richard Sohnius, Eyck Jentzsch, Wolf-Ekkehard Matzke \\ Cadence Design Systems, GmbH, Mozart str., 2, 85622, Feldkirchen, Germany \\ rsohnius@cadence.com,jentzsch@cadence.com,wolf@cadence.com \\ Vadim Ermolayev \\ Zaporozhye National Univ., 66, Zhukovskogo st., 69063, Zaporozhye, Ukraine \\ vadim@ermolayev.com
}

Keywords: design system, assessment, simulation, Produktiv+, PSI, performance, productivity, dynamic engineering design process, ontology, multi-agent system, microelectronics

\begin{abstract}
This position paper presents our work in assessing engineering design systems in the field of microelectronics with respect to their performance and, more specifically, to productivity. Current mainstream process assessment systems show deficiencies of the representation and analysis when dealing with dynamic, self-optimizing processes. To overcome this, a project called PRODUKTIV+ has been created with the goal to develop a new approach. This approach is to create a model of a design system and simulate the colaborative behavior of the involved engineers using a system of cooperating, intelligent software agents. The assessment of a design system is then done based on the detailed simulation results.
\end{abstract}

\section{INTRODUCTION}

It is well known that the design of microelectronic devices gets more and more complex ${ }^{1}$ (Moore 1965). To keep design time and cost of such devices in reasonable boundaries, the performance of the system carrying out designs needs to be increased. However, one can not improve what can not be measured. Thus, a reliable and comprehensible way to measure engineering design performance needs to be developed.

Applying mainstream workflow and project management tools has proven to be not suitable in the domain of engineering design. Therefore, we develop a new approach capable of modeling, simulating and assessing design systems. This approach is based on cooperating intelligent agents for simulation and acts as a decision support tool by allowing to measure productivity and other performance related indicators and by helping to

\footnotetext{
1 Moore's empiric law (interpretation): every 18 months the processing power (of the product) doubles while cost holds constant.
}

improve performance by analysing bottlenecks and weak spots of a design system.

\section{RELATED WORK}

A considerable weakness of mainstream process modeling approaches is the consequence of their strength. Indeed, the majority of process modeling frameworks provides sophisticated means to describe a rich variety of process structures (vdAalst et al 2003). They do it in a rigorous, but rather static manner. Unfortunately, this fits only to well-defined processes, for example in manufacturing. However, design processes are of a different kind: They “... are frequently chaotic and non-linear, and have not been well served by project management or workflow tools” (Neal, Smith \& Butler 2001).

Commercial offerings like those of Numetrics Management Systems, Inc ${ }^{2}$ provide a benchmarking service to assess development capabilities and analyze the design cycle to identify which phases are

\footnotetext{
${ }^{2}$ http://www.numetrics.com
} 
having the greatest impact on productivity and cycle time performance. Users of this service receive a quantitative assessment of their productivity, cycle time performance, throughput, design process quality, schedule performance and reuse strategy effectiveness. However, Numetrix's model of a design process is based on the black box principle. Their evaluation therefore uses only integral characteristics. The weak point is that such an approach does not allow "what-if" analysis reliably.

Other approaches like those described in (Jacome 1993), (Sutton 1997), (Gilmore \& Kloul 2005), (Heller, Schleicher \& Westfechtel 2003) take into account the nature of continuous process evolution but neglect the source of this evolution: the human beings involved in the process and pro-actively driving it.

The approach presented in this position paper is multidisciplinary and is aimed to integrate the strong points of the mentioned R\&D findings. It employs the minimal necessary sub-set of business process modelling primitives. Its strength lies in the fact that it is focused on the dynamic and stochastic character of design processes and uses AI planning and agentbased approach in software implementation. Thereby, it considers the impact of a human designer and a design team on the process flow and its productivity as very substantial.

The rest of the paper is structured as follows: section 3 reports about the project frame while section 4 describes the approach itself. Section 5 outlines potential use cases followed by the conclusion and outlook in section 6 .

\section{PROJECT FRAME}

To develop the approach and a prototype implementation a joint research project between AMD Saxony LLC, Robert Bosch GmbH, Infineon Technologies AG and Cadence Design Systems $\mathrm{GmbH}$ has been established. It is called PRODUKTIV ${ }^{3}$ and is partially funded by the German government. Additionally scientific institutes are incorporated as subcontractors namely IMS, OFFIS, metheval Jena, and Fraunhofer IIS. The project uses results of the $\mathrm{PSI}^{4}$ project (Matzke 2005).

The objective of PRODUKTIV+ is to develop a comprehensible model and reference system for

\footnotetext{
${ }^{3}$ http://www.edacentrum.de/produktivplus

${ }^{4}$ Performance Simulation Initiative, internal research project of Cadence.
}

acquiring performance indicators of microelectronic design systems executing design processes. Since those are highly creative and human driven processes the approach puts strong emphasis on the designers' abilities and their interaction with the design system and processes. Due to the dynamic nature of design processes we adopted simulation as the means of gaining detailed data. This allows not only to analyse data retrospectively but also to predict the behavior and performance.

\section{ASSESSMENT APPROACH}

The approach comprises three steps. It uses the suite of ontologies, the set of metrics, and dynamic behavioral models (Figure 1). The description of the ontology is given in the section 4.1, the metrics and the behavioral models are described in section and 4.3.

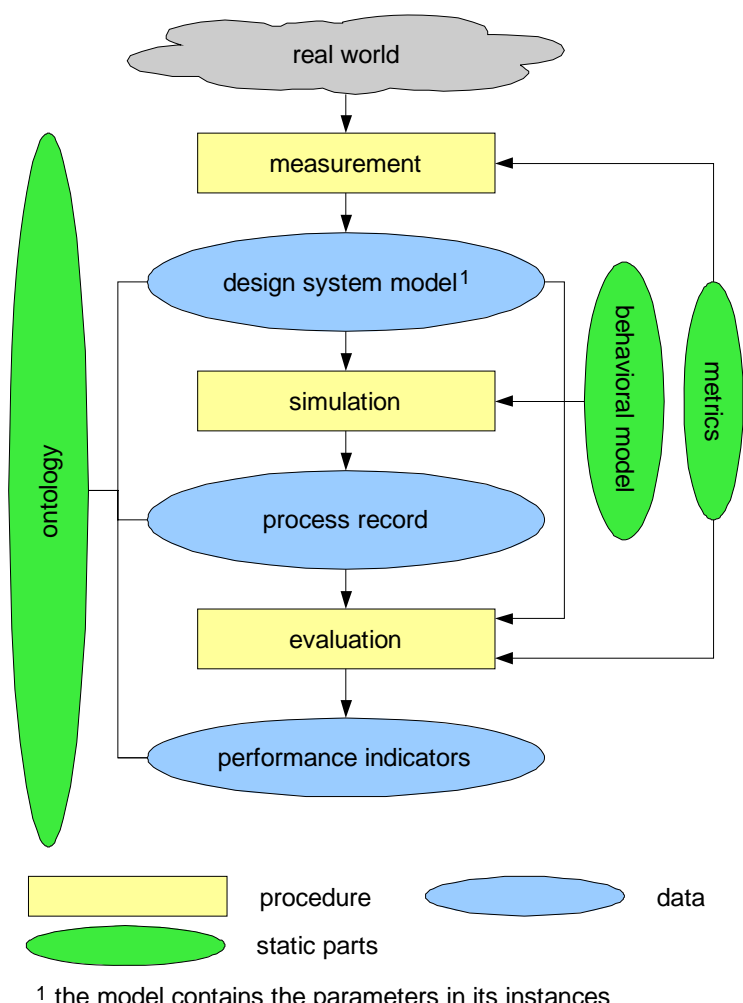

Figure 1: The PRODUKTIV+ Flow.

As in any assessment process, the first step is collecting information. But unlike other approaches, this information is more tied to the elements of the design system. Therefore it is more detailed and not 
directly related to the performance indicators. This data is used to fill the model of the design system with its parameters. The model consists of several parts: an ontology specifying the elements, their properties and relationships, a behavioral specification describing the algorithmic parts of the model and the metrics specification.

At the next step, the design system with the given parameters is simulated and the parameters are adjusted so that the results fit observable results and properties. With a simulation model calibrated this way it possible to gain very detailed data about the process (the process record).

In the last step this record together with the initial data can be analyzed and evaluated resulting in the desired performance indicators and measures.

\subsection{Model}

A characteristic feature of the model of a design process in PRODUKTIV+ is the way it addresses the dynamic properties of a process attempting to come closer to the reality in this application domain. The aim is to model processes which are selfadjusting, self-configuring, and self-optimizing at run time (Ermolayev et al 2006a). The model is based on the notion of a Dynamic Engineering Design Process (DEDP). One of the central points in modeling a DEDP is to adequately represent a designer as a locus of a goal-directed behavior and a design team as a dynamic social structure. A software agent can be naturally used as an appropriate model for a designer and a multi-agent system (MAS) as a model for an adaptable social structure like a design team. This is why PRODUKTIV+ uses agent-oriented models in its framework.

A DEDP is understood as a weakly defined engineering design workflow. It aims to achieve its goal (the design artifact comprising a certain set of its representations) in an optimized way in the terms of result quality and gained performance. The following entities are involved in the process: actors, who form design teams and collaboratively do the work in the flow; activities which are the atomic parts of a workflow defined by the technology used in the house; tasks which are the representations of the hierarchical clusters of activities; and design artifacts which are the results of engineering design activities. Hence, activities are defined statically and form the "basket" of activities, are uniformly understood and used by any actor and, therefore, may be considered generic. Another static shared "basket" is the one of generic tasks (below). Other elements may only "become apparent" at run time.
An executable activity differs from a generic one by having particular associations to the assigned actor and the developed design artifact.

Tasks are also distinguished as generic and performed ones. A generic task is a shared static template defining a typical transformation of a design artifact. A performed task is subjectively dynamic because of its relationship to the specific actor who manages the task assigned at run time.

A task is the model of the emerging hierarchical structure of a DEDP or the part of a DEDP. It may contain tasks or wrap a single activity. The main purpose of a task is to arrange the assignment of its sub-tasks or the execution of the wrapped activity. By formalizing the above we denote the model of dynamic cascade decomposition of tasks and, ultimately, of a DEDP.

The number of activity loops is not defined in advance. It depends on the quality checks at intermediate steps. Changing the number of activity loops may cause the changes in its duration. In turn, it may cause the delays of the dependent tasks and activities with associated penalties for, e.g., deadline violation.

The duration of activity execution is not defined in advance either. Different actors possess different capacities to be spent for an activity at a certain time. They may perform the same activity with different efficiency depending on their ability. An activity may remain idle while waiting until the preconditions have been triggered. Idle state duration can't be computed in advance because the preconditions may be formed by the other activities executed by other actors.

Mentioned factors provide certain degrees of freedom $^{5}$ in DEDP planning, re-planning, scheduling, re-scheduling, and execution. A DEDP is never rigidly planned before it starts. The decisions on how to continue its execution are taken each time it reaches a certain state in the state space. These decisions are taken by the design team members through reaching agreements in negotiations (Ermolayev \& Keberle 2006).

The described model has been formalized in the suite of PRODUKTIV+ ontologies v.1.0 extending PSI Ontologies suite v.1.6 (Ermolayev et al 2006b). These ontologies have been used to formalize the test cases (Sohnius et al 2006) and feed the simulation prototype (Samoylov et al 2006).

\footnotetext{
${ }^{5}$ It should be noted here that this freedom implies more complications in planning, scheduling and the necessity to deal with finer grained DEDP model.
} 


\subsection{Measurement}

In order to be able to do a simulation, detailed information on the design team, tools, resources, and of course the product to be designed are necessary. Furthermore, as much information as possible on the course of the project is required to be able to calibrate the simulation.

Data on the first test cases has been collected manually and with some semi-automated export functionality (Sohnius et al 2006). Experimental approaches on gaining large amounts of data automatically from process logs and license servers are currently tested. Furthermore, a first GUI-based software tool allowing easier and more comfortable collection of data that cannot be extracted automatically has been developed.

Complete testcases collected so far include two simple fictive testcase on a digital and an analog design, one fictive testcase of a mixed signal MP3 player chip, and one real world case of a digital design (Sohnius et al 2006).

\subsection{Simulation}

The next step is to simulate the course of the project. The goal of the simulation is to achieve a selfregulating system that creates reasonable project plans including schedules and is capable of proactively reacting to external influences as a team of designers would do. The simulation of the execution of the plan is based on the estimations of the duration and achieved quality of each activity. The natural way of simulating such a group of cooperating humans is a system of cooperating software agents - a multi agent system (MAS) (Samoylov et al 2006). Every agent represents an engineer and they collaborate to accomplish the project. Whereby the roles an agent can play are not restricted to engineering and design work but also include managing activities like a project or task manager.

First, the MAS has to create a plan - meaning a work breakdown structure, a resource assignment, and a schedule. Whereby, as mentioned before, the WBS is created by assembling the known activities to reach the project goal. A partial view of the resulting activity graph is shown in Figure 2. Bright activities and states were found to be a less optimal choice and have been removed automatically. At the next step, the execution of this plan is simulated and the MAS reacts dynamically to any influence disturbing the plan (section 5.3).

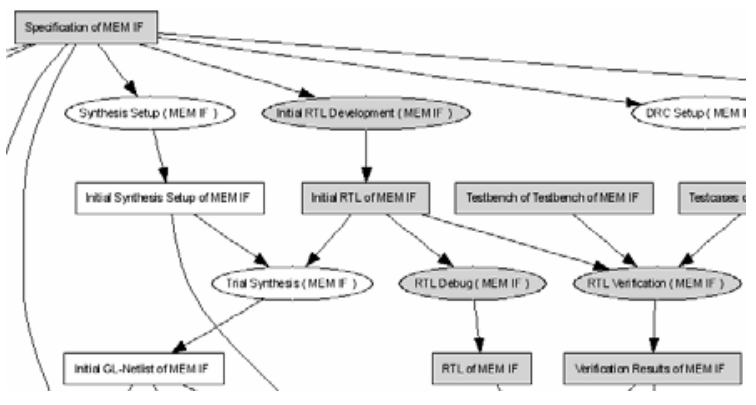

Figure 2: Partial WBS Graph.

Before the simulation can be used to gain the desired data, it needs to be calibrated. This is done by running the simulation with the parameter values collected in advance and comparing the results to the course of the real project. Experience shows that the collected data usually contains mistakes and inconsistencies which implies that the simulation is unable to complete or completes with senseless results. Another reason for larger deviations is the existence of external influences which occured in the real project but were forgotten in the simulation setup - e.g. events which result in changes of the objectives, of the staff or of the available resources in the course of the project. One more reason might be that not all company policies, restricting the engineers' choices, were formalized. The parameters must be corrected and completed until the simulation completes with results close to the original project.

Once the simulation is calibrated, the record of the simulation can be used to gain very detailed information on the course of the project. While this information is of course only a "most likely" estimation, it is still information that would require tremendous effort to collect in the real world or is even impossible or forbidden to acquire. Furthermore, the very same system can be used for predictive simulations and therefore allows what-if analyses (section 5.2).

So far, an initial simulation with the most important features has been implemented based on the first model (Samoylov et al 2006). It has been tested using the aforementioned test cases and showed promising results: The system came up with a consistent and complete plan. It decomposed the design correctly and chose appropriate activities to reach the project goal. Furthermore, it proposed a resource assignment and a schedule. All of that was reviewed by the original project leader and considered reasonable and acceptable. The simulation also reacted correctly to changes to the 
parameters like changes in the staff, the structure or the complexity of the design.

\section{ASSESSMENT}

Using the collected information supplemented with the records of the simulation several different evaluations and assessments are possible.

\subsection{Performance Assessment}

Our foremost goal is the assessment of the performance of a design system. For example, to assess productivity, we compare the inputs (costs) with the output which is computed using the complexity of the design, its achieved quality, and the level of abstraction crossed.

Other important indicators are development time respectively time-to-market, development cost, utilization, and more.

Thanks to the high level of detail available, we can do that not only for the whole design but also for certain design phases and/or certain parts of the design. Comparisons of these numbers can be used to identify "weak spots" where there is more potential for the improvement of the gained performance.

\subsection{What-If Analysis}

What-if analysis is the approach to make the mentioned "weak spots" more efficient (or "performative"). Initial experiments with the prototype simulation tool (Samoylov et al 2006) proved to be promising in that. It is possible to change some of the inputs of the simulation and iterate it to see which effect the changes will have. Such an analysis allows for ROI assessments like "how much do I gain if I buy this new computer?" and more complex short-term/mid-term analyses like "how much do I loose in the beginning if I switch to that new tool suite?", "when will I have a break even?" (with respect to productivity) and "how much do it gain once the tools are fully integrated?”.

These analyses can also help with project related decisions like "which technology to use?" or "design the part in house or buy an available $\mathrm{IP}^{6}$ ?”.

Another potential use case is feasibility studies. Once the tool is fed with the information about the

6 IP stands for "Intellectual Property" and denotes predesigned modules which can be inserted into designs. design system, one can also make estimations about the feasibility of different kinds of design. The simulation might either fail with the agents reporting that they were unable to come up with a reasonable plan due to the lack of some skills or tools or in the better case, they will present a rough estimate of the project with respect to the company's specific profile. This estimate can then be analyzed like a normal replay and weak spots and critical issues can be dealt with upfront.

\subsection{Random Simulation}

Having an average estimate of the course of a future project is nice but it does not tell how safe or risky this plan is. In real life, there are too many influences on a project to give a $100 \%$ precise prediction. Hense, by introducing a similar uncertainty into the simulation, varying activity lengths and adding random events like specification changes and change of staff, it is possible to estimate how stable the plan is regarding these disturbing influences. In order to do that, a whole series of random simulation has to be performed (e.g. using Monte Carlo method) and the results have to be analyzed statistically.

A small fraction of the result of such a random simulation run can be seen in Figure 3. The brighter bars show the planned execution time and the darker ones the time in the random simulation.

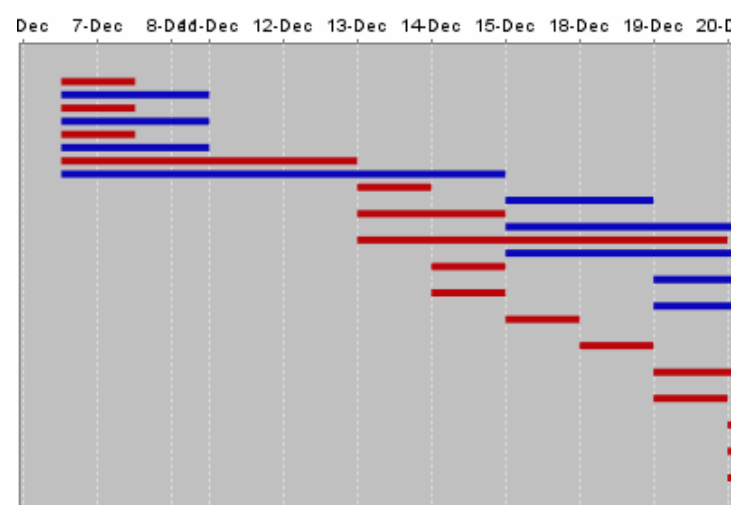

Figure 3: Simulation Result.

\subsection{Quality Management}

Last but not least, the system can help with quality management. The simulation also includes quality estimations and allows therefore answering questions like: "what quality will my product most 
likely have?”, "where can get the most improvement in quality?” or "where can I save some time or costs with minimal impact on quality?"

\subsection{Work done so far}

Some basic indicators like development time, total effort, utilization, and others have been implemented. More complex evaluations are not implemented yet as some of their inputs are not fully present at this time.

Simulations of the same project with different parameters have been conducted and the results have been compared using the aforementioned indicators. The results matched the expectations.

Furthermore, some simple randomized simulations have also been conducted to show the feasibility of such an analysis.

\section{CONCLUSIONS}

While the development and implementation of some important aspects is not complete yet, the results are already promising. We have shown that the approach in general is feasible.

Next steps obviously include the completion of the model and its implementation, as well as improving the pool of data to allow for better evaluation.

Crucial to the success of this approach will be the amount of data required to get reasonable results and the effort that has to be spent in order to gain this data. A final answer to that question can only be given after the model is complete, implemented, fine tuned, and analyzed and the efforts on automatic data gathering are also completed.

\section{REFERENCES}

Ermolayev, V., Jentzsch, E., Karsayev, O., Keberle, N., Matzke, W.-E., Samoylov, V., Sohnius, R. (2006a). An Agent-Oriented Model of a Dynamic Engineering Design Process. In: Kolp, M.et al. (Eds.): AgentOriented Information Systems III. 7th Int. Bi-Conf. Workshop, AOIS 2005, Utrecht, Netherlands, Jul. 26, 2005, and Klagenfurt, Austria, Oct. 27, 2005. Revised Selected Papers, 168-183

Ermolayev, V., Jentzsch, E., Keberle, N., Sohnius, R. (2006b). Performance Simulation Initiative. The Family of PSI Ontologies v.1.6. Reference Specification. Technical Report PSI-ONTO-TR-20064, 26.07.2006, VCAD EMEA Cadence Design Systems, GmbH, 78 p.
Ermolayev, V., Keberle, N., (2006). A Generic Ontology of Rational Negotiation. In: Karagiannis, D., Mayr, H.C. (Eds.): Information Systems Technology and its Applications. 5-th Int Conf ISTA'2006, May 30 - 31, 2006, Klagenfurt, Austria, 51-66

Gilmore, S., Kloul, L. (2005). A Unified Tool for Performance Modelling and Prediction, Reliability Engineering and System Safety, Elsevier Science, vol.89, n.1, pp:17-32, July 2005

Heller, M., Schleicher, A., Westfechtel, B. (2003). A Management System for Evolving Development Processes. Integrated Design and Process Technology, IDPT-2003

Jacome, M. F. (1993). Design Process Planning and Management for CAD Frameworks. Dissertation at the Carnegie Mellon University, Pittsburgh, Pennsylvania 1993

Matzke, W.-E. (2005). Engineering Design Performance Management - from Alchemy to Science through ISTa. In: Kaschek, R., Mayr, H.C., Liddle, S. (Eds.) Proc. $4^{\text {th }}$ Int. Conf on Information Systems Technology and its Applications (ISTA'2005), 23-25 May 2005, Palmerston North, New Zealand, 154-179, 2005

Moore, G.E.(1965). Cramming more components onto integrated circuits. Electronics, Vol 38, No 8, April 19, 1965, pp. 114-117

Neal, D., Smith, H. and Butler, D. (2001). The evolution of business processes from description to data to smart executable code - is this the future of systems integration and collaborative commerce? Research Services Journal: March 2001, 39-49

Sohnius, R., Ermolayev, V., Jentzsch, E., Keberle, N., Matzke, W.-E., Samoylov, V. (2006). Managing Concurrent Engineering Design Processes and Associated Knowledge. In: Ghodous, P., Dieng-Kuntz, R., and Loureiro, G. (Eds.): Leading the Web in Concurrent Engineering. Proc. 13th ISPE Int Conf on Concurrent Engineering: Research and Applications, 18 - 22 Sept., Antibes, French Riviera, IOS Press, Series: Frontiers in AI and Applications, Vol. 143, pp. 198-205, 2006

Samoylov, V., Gorodetsky, V., Ermolayev, V., Jentzsch, E., Karsayev, O., Keberle, N., Matzke, W.-E., and Sohinus, R. (2006). Agent-based Prototype of the Dynamic Engineering Design Process Performance Management System (DEDP-PMS). In: Brewka, G., Coradeschi, S., Massa , P., Perini, A., Tatar, M., Traverso, P. (Eds.) ECAI 2006 Systems Demonstrations . ECAI 2006, Riva del Garda, Italy , Aug. 28 - Sep. 1 , p. $37-38$

Sutton, P.R. (1997). A Framework and Discipline Independent Approach to Design System Management. Dissertation at the Carnegie Mellon University, Pittsburgh, Pennsylvania, 1997 